\title{
ArCo: An R package to Estimate Artificial Counterfactuals
}

\author{
by Yuri R. Fonseca, Ricardo P. Masini, Marcelo C. Medeiros and Gabriel F. R. Vasconcelos
}

\begin{abstract}
In this paper we introduce the ArCo package for $\mathrm{R}$ which consists of a set of functions to implement the the Artificial Counterfactual (ArCo) methodology to estimate causal effects of an intervention (treatment) on aggregated data and when a control group is not necessarily available. The ArCo method is a two-step procedure, where in the first stage a counterfactual is estimated from a large panel of time series from a pool of untreated peers. In the second-stage, the average treatment effect over the post-intervention sample is computed. Standard inferential procedures are available. The package is illustrated with both simulated and real datasets.
\end{abstract}

\section{Introduction}

In this paper an $\mathrm{R}$ package to conduct counterfactual analysis by the Artificial Counterfactual (ArCo) method is introduced. The ArCo methodology is a flexible and easy-to-implement method to estimate the causal effects of an intervention on a single treated unit and when a control group is not readily available. The procedure consists of two steps where, in the first stage, a counterfactual is estimated on the basis of a large-dimensional set of variables from a pool of untreated units ("donors pool"). In the second stage, the average intervention effect on a vector of variables from the treated unit is estimated. The method is robust to the presence of confounding effects, such as a global shock and can be seen as an extension of the Synthetic Control (SC) approach of Abadie and Gardeazabal (2003) and Abadie et al. (2011) and the panel factor (PF) model method put forward by Hsiao et al. (2012).

Causality and counterfactual analysis are topics of major importance in Social Sciences, Economics, Medicine, Psychology, etc. Routinely, causal statements with respect to a given intervention (or treatment) depend on the construction of counterfactuals, which are estimated from the outcomes of a similar group of individuals not affected by the intervention. Nevertheless, reaching final causeand-effect conclusions is a very challenging task as a consequence of the difficulties in finding reliable sources of exogenous variation. In microeconometrics there have been major advances in the literature and the estimation of treatment effects is now part of the current toolbox of applied researchers. On the other hand, when there is not a natural control group and there is a single treated unit, which is usually the case when handling, for example, macro (aggregated) data, the econometric tools have evolved at a slower pace. However, in the recent past, some authors have proposed new techniques inspired partially by the developments in microeconometrics that are able, under some assumptions, to estimate counterfactuals with aggregate data. The ArCo method fits into this exciting literature. See, for example, Athey and Imbens (2016a) or Athey and Imbens (2016b) for recent reviews.

\section{Overview}

All the econometric analysis conducted with the ArCo method is on the time dimension and it consists of the following key ingredients: the definition of the intervention and the intervention time, the units of interest (treated and untreated units) and the observed variables for each unit. Units are, for example, municipalities, states, regions, countries, products, firms, stores, etc. The intervention occurs in one of these units starting in a given point in time and does not affect the other units. Interventions could be war out-breaks, terrorist attacks, policy changes, enforcement of new laws, natural disasters, new prices, among many others. Each unit is described by at least one variable. For example, if we are dealing with countries the variables may be GDP and inflation and for companies they could be profit and revenue. For products the variables could be prices and sold quantities. All variables are observed along a period of time (days, months, years, for example).

The ArCo method is a two-step procedure. In the first step, the data before the occurrence of the intervention is used to estimate a multivariate time-series regression model relating the variables in the treated unit (dependent variables) with only the variables belonging to the untreated peers (explanatory variables). In the second step, the counterfactual is constructed by extrapolating the estimated model with data after the intervention. The estimated effect is the time-series average of the difference between the actual data and the counterfactual. It is important to stress that the model is conditioned on data from the untreated peers only and the validity of the method is based on two key assumptions:

1. The peers are not affected by the intervention; 


\section{The data is trend-stationary.}

The first assumption is common in the literature and has been also assumed by Abadie and Gardeazabal (2003) and Hsiao et al. (2012). On the other hand, the trend-stationarity assumption has been dangerously ignored in the literature. Carvalho et al. (2016a) investigate the consequences of applying the ArCo, SC or PF methods when the data are integrated processes of order 1 . They find that without a cointegrating relationship (spurious case) the estimator of the effects of the intervention diverges, resulting in the rejection of the null hypothesis of no effect regardless of its existence with probability approaching 1 . For the case when one cointegrating relationship exists, the estimator is $\sqrt{T}$-consistent for the intervention effect albeit with a non-standard distribution. Nevertheless, even in this case, the test of no intervention effect is extremely oversized if nonstationarity is ignored. When there is a drift in the data generating processes, the estimator for both cases (cointegrated and spurious) either diverges or is not well defined asymptotically. The authors thus strongly recommend the use of first-differenced data to avoid spurious results in case of integrated data. Furthermore, heterogeneous, possibly nonlinear, deterministic time trends are allowed among units. See also Ferman and Pinto (2016) for a similar discussion.

High-dimensionality is relevant when the number of parameters to be estimated is large compared to the actual sample size. This can happen either when the number of peers and/or the number of variables for each peer is large or when the sample size is too small. In Carvalho et al. (2016b) the authors consider a linear model estimated by the Least Absolute Selection and Shrinkage Operator (LASSO) proposed by Tibshirani (1996). However, in the R package we leave the choice of the conditioning model to the user. LASSO regressions (and extensions), regression trees and random forests, boosted trees, neural networks, splines, factor models, are some possible examples of models to be estimated in the first step. The results in Carvalho et al. (2016b) are derived under asymptotic limits on the time dimension $(T)$. However, the authors allow the number of peers $(n)$ and the number of observed variables for each peer to grow as a function of $T$.

\section{A Glimpse on the Literature and Comparison with Other Methods}

The ArCo approach nests the PF method proposed by Hsiao et al. (2012) and can been seen as a generalization of the SC approach in the same lines as discussed by Doudchenko and Imbens (2016) and Ferman and Pinto (2016). It is also better suited than Difference-in-Differences (DiD) estimators for comparative case studies when there is a single treated unit and no similar control group is available, even after the inclusion of many control variables. Furthermore, the ArCo approach relaxes the stringent parallel trend restriction of the DiD methods.

Recently, Gobillon and Magnac (2016) generalize DiD estimators by considering a correctly specified linear panel model with strictly exogenous regressors and interactive fixed effects represented as a number of common factors with heterogeneous loadings. Their theoretical results rely on double asymptotics when both $T$ and $n$ go to infinity. The authors allow the common confounding factors to have nonlinear deterministic trends. The ArCo method differs from Gobillon and Magnac (2016) in several directions. First, as mentioned before, the model is not assumed to be correctly specified and there is no need to estimate the common factors. Consistent estimation of factors needs that both the time-series and the cross-section dimensions diverge to infinity and can be severely biased in small samples. The ArCo methodology requires only the time-series dimension to diverge. Furthermore, the regressors do not need to be strictly exogenous which is an unrealistic assumption in most applications with aggregate data. Heterogeneous nonlinear trends are also allowed but there is no need to estimate them (either explicitly or via common factors). Finally, as in the DiD case, the ArCo does not either require the number of treated units to grow or to have a reliable control group (after conditioning on covariates).

Although, both the ArCo and the SC methods construct a counterfactual as a function of observed variables from a pool of peers, the two approaches have important differences. First, the SC method relies on a convex combination of peers to construct the counterfactual which, as pointed out by Ferman and Pinto (2016), biases the estimator. The ArCo solution is a general, possibly nonlinear, function. Even in the case of linearity, the method does not impose any restriction on the parameters. For example, the restriction that weights in the SC methods are all positive seems a bit too strong. The SC method also requires an unrealistic identification assumption about the (perfect-)fit of the model in the pre-intervention period. Furthermore, the weights in the SC method are usually estimated using time averages of the observed variables for each peer. Therefore, all the time-series dynamics is removed and the weights are determined in a pure cross-sectional setting. In addition, the SC method was designed to evaluate the effects of the intervention on a single variable. In order to evaluate the effects in a vector of variables, the method has to be applied several times. The ArCo methodology can be directly applied to a vector of variables of interest. In addition, there is no formal inferential procedure for hypothesis testing in the SC method, whereas in the ArCo methodology, a simple, 
uniformly valid and standard test can be applied ${ }^{1}$. Finally, as discussed in Ferman et al. (2016), the SC method does not provide any guidance on how to select the variables which determine the optimal weights ${ }^{2}$.

\section{Framework}

This section aims to describe the mathematical notation and the key definitions of the ArCo methodology in a way that is compatible with the ArCo (Fonseca et al., 2017) package. For further details on statistical properties and theoretical results, see Carvalho et al. (2016b) and Carvalho et al. (2016a). Everything concerning the technique used to estimate the first-step model is left in a very general way as the ArCo package was developed to accept many different classes of models. Note, however, that the theory and the inference was developed for LASSO linear regressions.

- units: Indexed by $i=1, \ldots, n$. Units are, for example, municipalities, states, regions, countries, products, firms, stores, etc. The treatment occurs in one of these units and do not affect the others.

- variables: For each unit $i, i=1, \ldots, n$, and for every time period $t, t=1, \ldots, T$, we observe $q_{i} \geq 1$ variables. If the units are, for example, firms, the variables may be sales, income, profit, etc. We will refer to these variables as $z_{i t}=\left(z_{i t}^{1}, \ldots, z_{i t}^{q_{i}}\right)$.

- intervention: The intervention took place only in the treated unit at time $T_{0}=\lambda_{0} T$ with $\lambda_{0} \in(0,1)$.

Assume, without loss of generality, that the treated unit is the first one $(i=1)$. Furthermore, let $z_{1 t}^{(0)}$ and $z_{1 t}^{(1)}$ be the outcomes of the first unit under treatment and without treatment, respectively. Normally, we do not observe both outcomes simultaneously. Instead, we observe:

$$
z_{1 t}=D_{t} z_{1 t}^{(0)}+\left(1-D_{t}\right) z_{1 t}^{(1)}
$$

where $D_{t}$ assumes value 1 if the unit in under treatment at time $t$ and 0 otherwise.

The goal is to test the hypothesis on the effects of the intervention being statistically significant for $t \geq T_{0}$. The interventions are considered in the form

$$
y_{t}^{(1)}= \begin{cases}y_{1 t}^{(0)}, & t=1, \ldots, T_{0}-1 \\ y_{1 t}^{(0)}+\delta_{t}, & t=T_{0}, \ldots, T,\end{cases}
$$

where $\boldsymbol{y}_{t}^{(j)}=\boldsymbol{h}\left(\boldsymbol{z}_{1 t}^{j}\right) \in \mathbb{R}^{q}$ for $j \in\{0,1\}, \boldsymbol{h}(\cdot)$ is a measurable function of $\boldsymbol{z}_{1 t}$ and $\left\{\delta_{t}\right\}_{t=T_{0}}^{T}$ is a deterministic sequence. The function $h(\cdot)$ is very general and allows interventions on the mean, variance, covariance, etc. The ArCo method is concerned with the following hypothesis:

$$
\mathcal{H}_{0}: \Delta_{T}=\frac{1}{T-T_{0}+1} \sum_{t=T_{0}}^{T} \delta_{t}=0,
$$

where $\Delta_{t}$ is the average treatment effect over the treatment period.

We do not observe $\boldsymbol{y}_{t}^{(0)}$ for $t \geq T_{0}$. This quantity is the counterfactual, i.e., what would $\boldsymbol{y}_{t}$ have been in the absence of intervention. In order to proceed to the first step estimation of the ArCo, let $z_{0 t}=\left(z_{2 t}^{\prime}, \ldots, z_{n t}^{\prime}\right)^{\prime}$ and $Z_{0 t}=\left(z_{0 t}^{\prime}, \ldots, z_{0 t-p}^{\prime}\right)^{\prime}$ be the collection of all untreated units up to an arbitrary lag $p \geq 0$. The dimension of $\boldsymbol{Z}_{0 t}$ depends on the number of units, the number of lags and the number of variables per unit.

Consider the following model for $\boldsymbol{y}_{t}^{(0)}$

$$
y_{t}^{(0)}=\mathcal{M}_{t}+\boldsymbol{v}_{t},
$$

where $\mathbb{E}\left(\boldsymbol{v}_{t}\right)=\mathbf{0}$ and $\mathcal{M}_{t}=\mathcal{M}\left(\mathbf{Z}_{0 t}\right)$. Note that $\mathcal{M}$ is a measurable mapping in a sense that it does not need to be an explicit function. For example, one could assume a regression tree or a random forest structure for $\mathcal{M}_{t}$.

The first step of the ArCo method consists on estimating (4) using the first $T_{0}-1$ observations, given that for $t<T_{0}$ we have $\boldsymbol{y}_{t}=\boldsymbol{y}_{t}^{(0)}$. Set $T_{1}=T_{0}-1$ and $T_{2}=T-T_{0}+1$, then one can estimate

\footnotetext{
${ }^{1}$ The theoretical results in Carvalho et al. (2016b) were derived for the case of LASSO regression only but can be easily extended to other classes of models.

${ }^{2}$ Doudchenko and Imbens (2016) advocate the use of shrinkage methods to estimate the pre-intervention model but no theory is provided.
} 
$\widehat{\mathcal{M}}_{t, T_{1}}=\widehat{\mathcal{M}}_{T_{1}}\left(\mathbf{Z}_{0 t}\right)$ and use it to construct the counterfactual

$$
\widehat{y}_{t}^{(0)}= \begin{cases}y_{t}^{(0)}, & t=1, \ldots, T_{0}-1 \\ \widehat{\mathcal{M}}_{t, T_{1}}, & t=T_{0}, \ldots, T .\end{cases}
$$

Finally, the ArCo estimator is defined as

$$
\widehat{\boldsymbol{\Delta}}_{T}=\frac{1}{T-T_{0}+1} \sum_{t=T_{0}}^{T} \widehat{\delta}_{t},
$$

where $\widehat{\boldsymbol{\delta}}_{t}=\boldsymbol{y}_{t}-\widehat{\boldsymbol{y}}_{t}^{(0)}$ and $t=T_{0}, \ldots, T$.

As mentioned in the Introduction, the ArCo methodology is a two-step estimator where the first step consists on the estimation of $\mathcal{M}$ on the pre-intervention sample and in the second step we estimate $\widehat{\Delta}$, which is the average intervention impact.

\section{Hypothesis Testing}

Carvalho et al. (2016b) shows the conditions and results for the asymptotic normality of $\widehat{\Delta}_{T}$ and also, how to consistently estimate its covariance matrix, given by $\boldsymbol{\Omega}_{T}$. Therefore, one can obtain a confidence interval for a chosen significance level $\alpha$ given by

$$
\mathcal{I}_{j, \alpha}=\left[\widehat{\Delta}_{j, T} \pm \frac{\widehat{w}_{j}}{\sqrt{T}} \Phi^{-1}(1-\alpha / 2)\right],
$$

for each $j=1, \ldots, q$, where $\widehat{w}_{j}=\sqrt{\widehat{\Omega}_{j j}}$ and $\Phi^{-1}(\cdot)$ is the quantile function of a standard normal distribution.

One can also establish a hypothesis test given that $W_{t}=T \widehat{\Delta}_{T}^{\prime} \widehat{\mathbf{\Omega}}_{T}^{-1} \widehat{\boldsymbol{\Delta}}$ has a chi-square distribution with $q$ degrees of freedom.

The estimation of $\Omega$ may be a challenge not because of the ArCo itself, but because of the robust estimator we may choose. The ArCo package allows the user to choose between the methods below:

- Covariance matrix assuming the errors are independent and identically distributed;

- prewhitening using VAR models as in Andrews and Monahan (1992). The lag of the VAR may be chosen by the user or though information criterion;

- Newey and West (1987) (NW) covariance matrix with quadratic spectral, truncated, Bartlett, Parzen or Turkey-Hanning kernels; and

- the combination of NW and prewhitening where the NW covariance matrix is calculated on the residuals of the VAR.

\section{Unknown Intervention Time}

In many cases it is reasonable to assume that the intervention time is unknown. For example, although some new policy has started at a known time, its effects may have been anticipated due to rational expectations. Regardless the source of the uncertainty, what we need is to estimate $\lambda_{0}$ by adjusting the ArCo estimator to be a function of $\lambda, \widehat{\Delta}_{T}(\lambda)$.

Set $\Lambda=(\underline{\lambda}, \bar{\lambda})$ as a bound for $\lambda_{0}$ to avoid finite sample bias close to the boundaries and define $\|\cdot\|_{p}$ as the $\ell_{p}$ norm, then

$$
\widehat{\lambda}_{0, p}=\underset{\lambda \in \Lambda}{\arg \max } J_{T, p}(\lambda), \quad J_{T, p}=\left\|\widehat{\boldsymbol{\Delta}}_{T}(\lambda)\right\|_{p} .
$$

Thus, $\widehat{\lambda}_{0, p}$ is the one that maximizes the $\ell_{p}$ norm of the ArCo estimator. If $\Delta \neq 0$ then $\widehat{\lambda}_{0, p}=$ $\lambda_{0}+o_{p}(1)$. On the other hand, if $\boldsymbol{\Delta}=\mathbf{0}$ then $\widehat{\lambda}_{0, p}$ converges in probability to any $\lambda \in \Lambda$ with equal probability.

\section{Asymptotic Theory and Sample Size}

The inferential procedures for the ArCo method are asymptotic. However, simulation experiments show that the method works well in small samples (Carvalho et al., 2016b). It is intuitive to think that the only sample size that matters is the one before the intervention as it is the one used to estimate the 
model. However, for a given sample size $T$, it is desirable to have the intervention as close as possible to $0.5 T$ because the average treatment effect, $\Delta$, also depends on the period after the intervention. In practice, if the number of variables and units is not very big, 40 to 50 observations should be enough as we show in the Basque example when we compare the ArCo with the Synthetic Control approach.

The first step of the ArCo is a time-series estimation. Therefore, all the data must have the same periodicity (year, quarters, months, etc). The Synthetic Control kills the time-series dimension of the control variables because it uses only their average (or other statistic such as median). This is an important difference between the methods: the ArCo uses all the time-series information but it requires more data than the Synthetic Control because all series must be complete (without missing values) and in the same periodicity.

\section{Practical Example: the fitArCo Function}

The ArCo package has two datasets that were created using the data generation process (DGP) from the second simulation example in Carvalho et al. (2016b) and another dataset from the same article's empirical example. In this section we walk through the ArCo package using these datasets. All examples presented here are entirely reproducible using the same codes and datasets.

The DGP is given by:

$$
z_{i t}^{(0)}=0.5 A_{i} z_{i t-1}^{(0)}+\varepsilon_{i, t}, i=1, \ldots, n, t=1, \ldots, T,
$$

where the error has a factor structure $\varepsilon_{i, t}=\boldsymbol{\Lambda}_{i} f_{t}+\boldsymbol{\eta}_{i t}$ with $f_{t}=\left[1,(t / T), v_{t}\right], z_{i t} \in \mathbb{R}^{q}, \boldsymbol{A}_{i}(q \times q)$ is a diagonal matrix with elements between -1 and $1,\left\{v_{t}\right\}$ is a sequence of independent and normally (NID) distributed random variables with zero mean and unit variance, $\left\{\boldsymbol{\eta}_{i t}\right\}$ is a sequence of NID random vectors with zero mean and covariance matrix $(0.2)^{2} I_{n q}$. We set $T=100$.

\section{Simple example for one variable}

The two generated datasets are data.q1 (one variable and 20 units) and data.q2 (two variables and 6 units). We will start with data.q1, which can be loaded with the following code:

$>\operatorname{library}($ ArCo)
$>\operatorname{data}($ data.q1)

The data.q1 is a matrix with 100 observations of 20 columns. In the ArCo context each column is a unit, therefore, we have one variable from each of the 20 units from the data. The intervention took place at the first unit at $T_{0}=51$ by adding a constant of 0.628 , which is one standard deviation from the treated unit before the intervention. The series in question is plotted in Figure 1 with a vertical line at $t=T_{0}$.

$>\operatorname{plot}($ data.q1[,1], type $=" 1 ")$

$>$ abline $(v=51$, col $=2,1$ ty $=2)$

The main function of the ArCo package is the function fitArCo, which estimates the ArCo and provides the relevant statistics and outputs. The data must be supplied in a list of matrices, each matrix should have $T$ observations of one single variable for all units. Therefore, if one has $q$ variables, the list must have $q$ matrices. Since the data.q1 has only one variable, we will supply the function with a list with only one matrix.

The user can choose any model to estimate the first step of the ArCo as long as it is possible to write a compatible estimation function (argument $f n$ ) and forecasting function (argument $p$. $f n$ ). The estimation function must receive a matrix of explanatory variables $X$ and the response variable $y$ in a vector, and it should return the estimated model; the forecasting function receives the output from the estimation function and the argument newdata ${ }^{3}$. For the purposes of this example we will write a $\mathrm{fn}$ and a $p . f n$ that uses simple Ordinary Least Squares (OLS). The OLS estimation is the default model of the fitArCo function in case the user does not supply any functions.

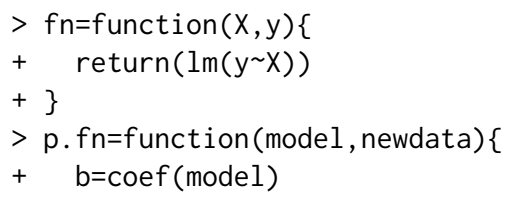

${ }^{3}$ The forecasting function is similar to most predict methods in $\mathrm{R}$, which receives a model object and the new data used on the prediction. 


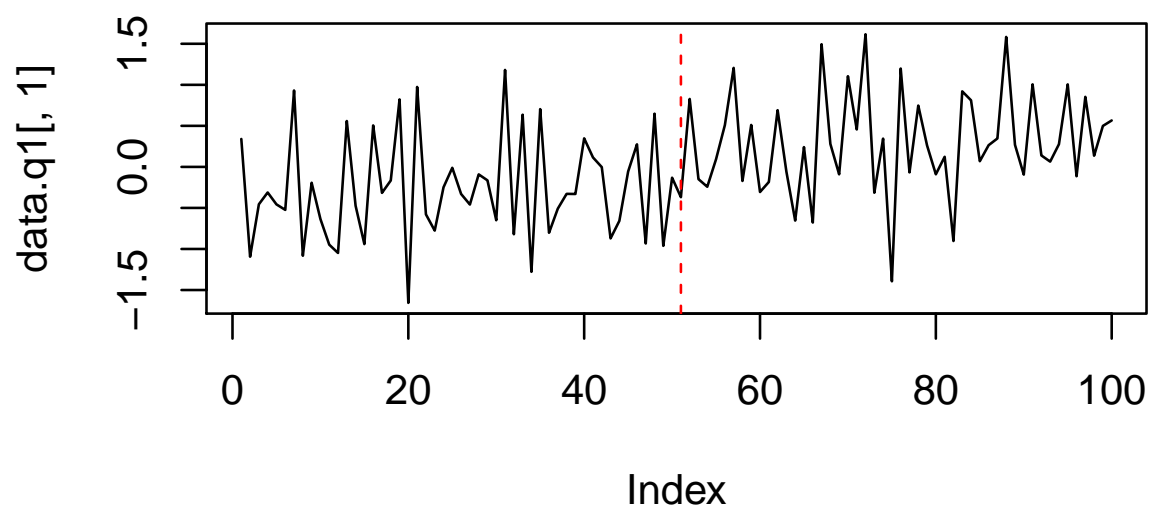

Figure 1: plot of data.q1 unit 1

$+\operatorname{return}(\operatorname{cbind}(1$, newdata) $\% * \%$ b)

+ \}

The ArCo can now be estimated:

$>\operatorname{ArCo}=f i t \operatorname{ArCo}($ data $=$ list (data.q1), $f n=f n, p . f n=p . f n$, treated. unit $=1$, to $=51$, VCOV.type $=$ "nw", VCOV.lag $=3$, prewhitening.kernel $=$ TRUE)

$>\operatorname{plot}($ ArCo, display. fitted=TRUE)

$>$ ArCo\$delta

Variable1 $0.4115779 \quad 0.51748890 .6233999$

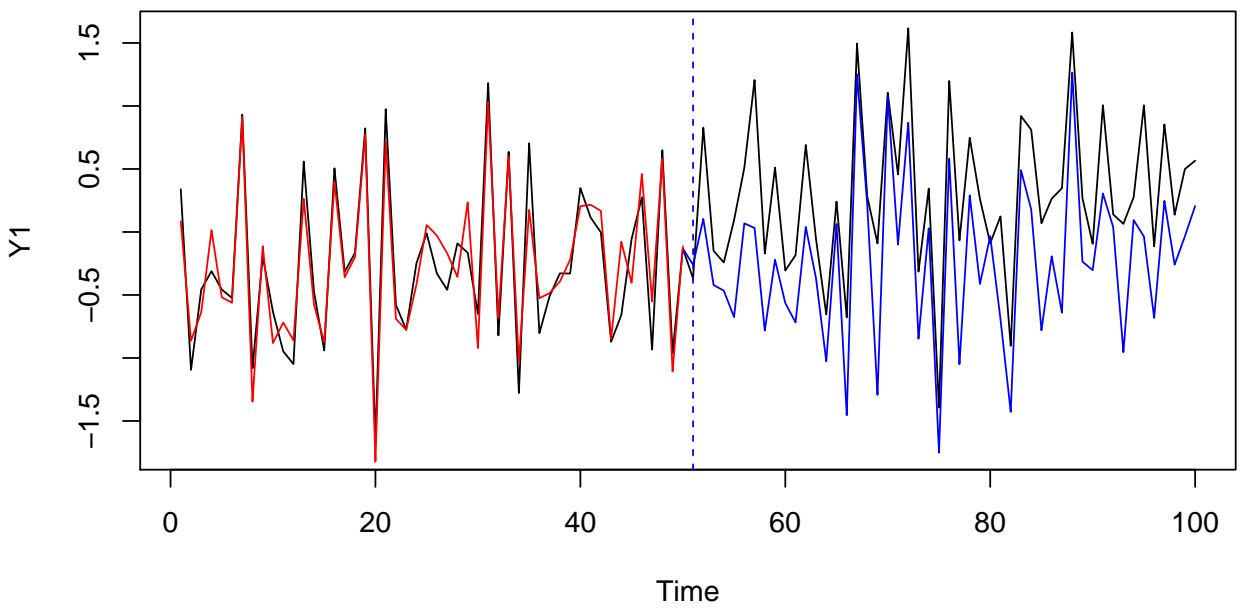

- Observed - Fitted - Counterfactual

Figure 2: plot of the counterfactual from data.q1 unit 1 
The fitArCo generates an object of class fitArCo with S3 method for plot $^{4}$ (Figure 2). Additionally, the user can chose from several types of robust covariance matrix estimations for $\boldsymbol{\Delta}_{T}$ such as Newey and West (1987) with prewhitening options from Andrews and Monahan (1992). The argument lag allows the inclusion of lags in the model and the argument Xreg accounts for exogenous controls. Finally, the $\$$ delta returns the $\Delta_{T}$ and its confidence bands for a significance level $\alpha$ chosen by the user and $\$ p$. value returns the ArCo $p$-value.

\section{Example for Two Variables and Bootstrap Inference}

In this section we show the ArCo with two variables and some additional features from the fitArCo function such as bootstrap estimation of the couterfactual confidence bands, which have the advantage that it can be calculated for any model chosen by the user.

If the argument boot.cf is set to TRUE the function will automatically perform block bootstrap replications of the counterfactual, the number of replications and the block length are controlled through the arguments $\mathrm{R}$ and 1 respectively. The bootstrap procedure uses the package boot (Canty and Ripley, 2015) and is described as follows:

1. For $t<T_{0}$ prepare the data in a way that the vector $y_{1}$ contains the response variable and a matrix $X_{1}$ contains all regressors;

2. sample $T_{0}-1$ - lag observations from $\left\{\boldsymbol{y}_{1}^{\prime}, \boldsymbol{X}_{1}\right\}$ using blocks of length $l$ generating the bootstrap sample $\left\{\boldsymbol{y}_{1}^{\prime}, \boldsymbol{X}_{1}\right\}^{r}$;

3. estimate the model on $\left\{\boldsymbol{y}_{1}^{\prime}, \boldsymbol{X}_{1}\right\}^{r}$;

4. for $t \geq T_{0}$, compute the forecast with the model estimated on step 3 using $X_{2}$ as the new data;

5. repeat steps $2-4 R$ times to obtain the counterfactual bootstrap distribution.

The data.q2 dateset has two variables for 6 units. The number of observations is 100 and the treatment of adding up one standard deviation ( 0.84 for the first variable and 0.511 for the second variable) took place at $T_{0}=51$ on the first unit.

The following example estimates the ArCo using LASSO with the glmnet (Friedman et al., 2010). The function $\mathrm{fn}$ is $\mathrm{cv}$.glmnet the function $\mathrm{p} . \mathrm{fn}$ is predict ${ }^{5}$. The function $\mathrm{cv}$.glmnet chooses the regularization parameter $\lambda$ using cross-validation.

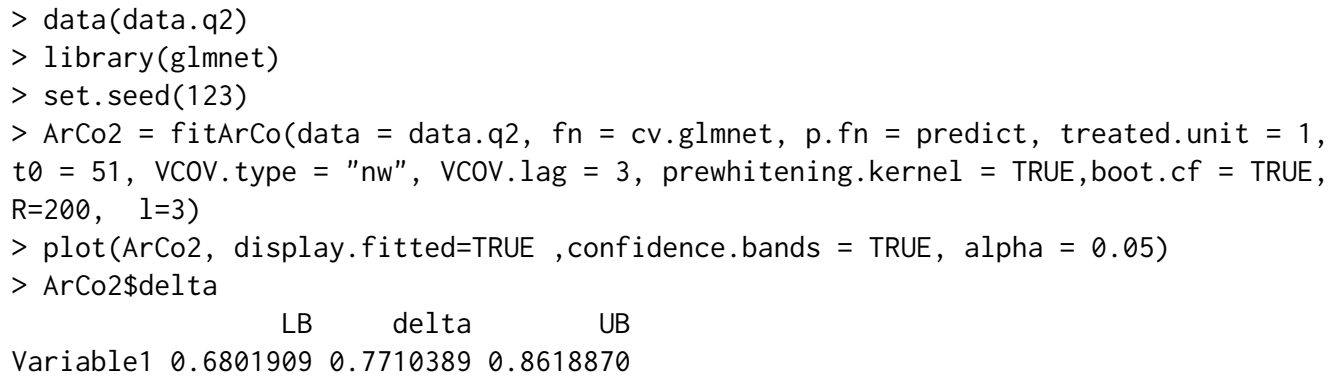

Variable2 $0.4225233 \quad 0.5071188 \quad 0.5917144$

Figure 3 shows the counterfactual with 95\% confidence bands. The red line shows if the chosen model has an acceptable performance in-sample. Moreover, the \$delta shows that the treatment was different from zero on both variables and the point value of $\widehat{\Delta}_{T}$ is close the value used to generate the data ( 0.84 and 0.511 for variables 1 and 2 respectively).

\section{Practical Advice on the Use of the fitArCo Function}

A potential issue for the practitioner is the choice of the function used to estimate the model in the first step of the $\operatorname{ArCo}(f n)$ method and the corresponding prediction function $(p . f n)$. The default is to estimate the ArCo using OLS because it is a simple procedure and require no extra packages. However, the most recommended model approach is to use LASSO to estimate a linear model due to its good properties in high-dimensional settings and, more importantly, because most the asymptotic theory for the ArCo method was developed for LASSO (high-dimensions) and OLS (low-dimensions). Nevertheless, the user is free to use any other models such as Random Forest, Boosted Trees, Neural

\footnotetext{
${ }^{4}$ The plot.fitArCo function will automatically display legends if the argument display.fitted is set to TRUE. For those who use RStudio, watch out for the figure margins, especially in cases where $q>1$ and the user wished to display multiple plots. If necessary use $\times 11($ ) to plot in a new window.

${ }^{5}$ Both functions are already on the format required by the fitArCo.
} 

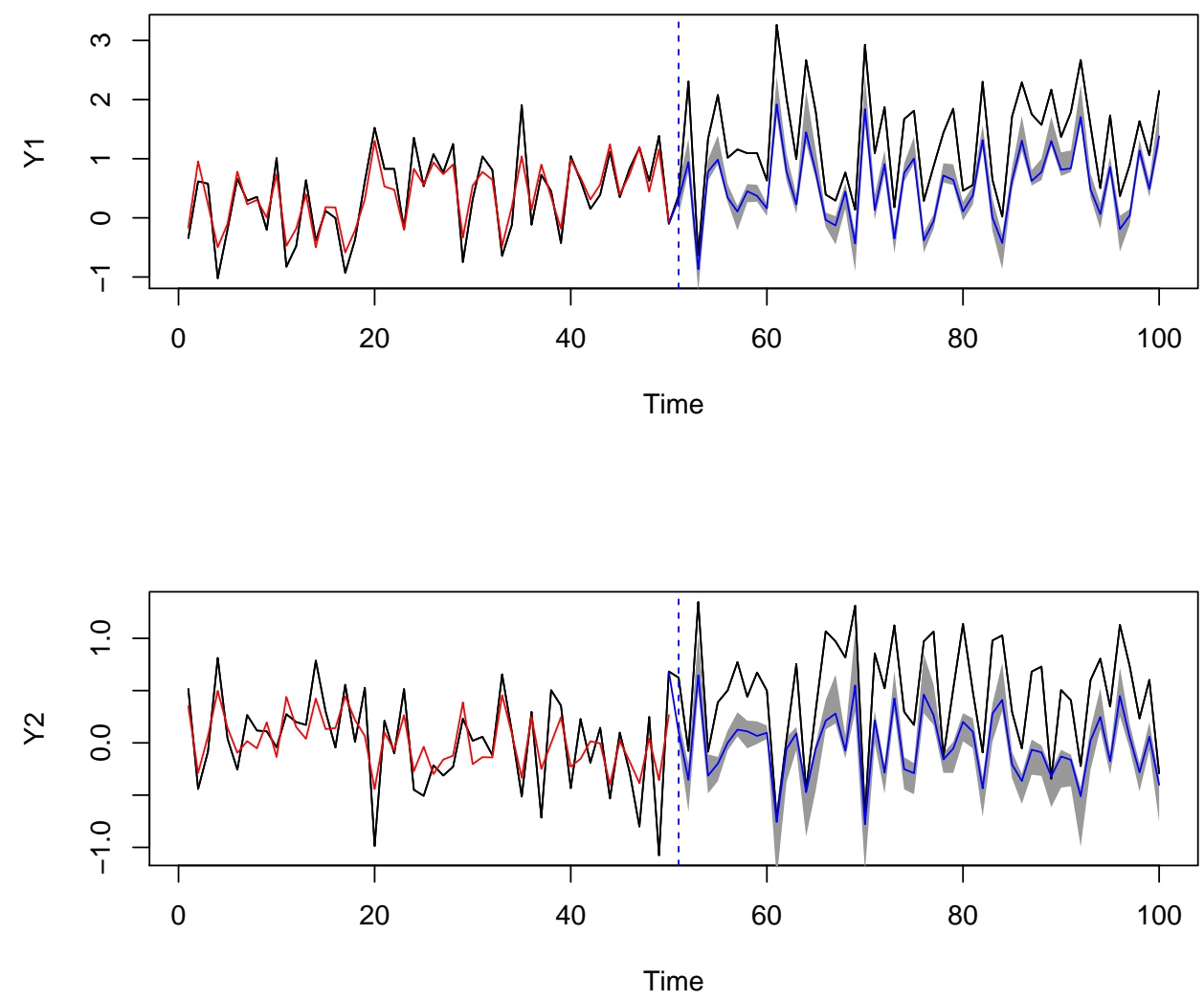

- Observed - Fitted - Counterfactual

Figure 3: plot of the counterfactual from data.q2 unit 1, variables 1 and 2, with bootstrap confidence bands. 
Networks, Factor Models, etc. In these cases, the user should be aware that the inference on the average treatment effect may not be valid and should only be used as an approximation. We recommend doing some simulation tests under the null hypothesis (no treatment) and under the alternative hypothesis (treatment different from zero) if the user chooses to use an estimation approach different from LASSO and OLS ${ }^{6}$.

The fitArCo accepts extra arguments to be passed to the fn function. This is not particularly useful for LASSO or OLS, but if the user chooses models that require tuning (Boosted Trees, Random Forest, etc) extra arguments may be required.

The fitArCo function returns all the inference regarding the average treatment effect (confidence intervals and $p$-values). We included several built-in methods in case a robust covariance matrix is required. We recommend the user to test the first-stage residuals for autocorrelation and heteroskedasticity. If these features are present in the residuals the most common solution is to use Newey-West (VCOV.type="nw") with Quadratic Spectral kernel (default). The optimal lag for the covariance matrix is calculated automatically but the user may force it through the argument VCov. lag.

The entire list of what the function fitArCo function returns is:

- cf: $T_{2} \times q$ matrix of the estimated counterfactual.

- fitted.values: $\left(T_{1}-\operatorname{lag}\right) \times q$ matrix with the fitted values for the pre-treatment period.

- model: A list with $q$ estimated models, one for each variable. Each model is the exact output of the $f n$ function.

- delta: The delta statistics for each variable and its confidence interval.

- p.value: The ArCo p-value.

- data: The data used.

- t0: The intervention period used.

- treated.unit: The treated unit used.

- boot.cf: A list with the bootstrap result (boot.cf=TRUE) or logical FALSE (boot.cf=FALSE). In the first case, each element in the list refers to one bootstrap replication of the counterfactual, i.e. the list length is $\mathrm{R}$.

- call: The matched call.

\section{The estimate_t0 Function}

We mentioned earlier that in many cases the exact intervention period might be unknown, but it can be estimated using the maximization of the norm of $\Delta_{T}(\lambda)$. This is precisely what the function estimate_t 0 does. The example below uses the dataset data.q2 and LASSO to estimate the model as in the previous example. The arguments start and end determine the range of $\lambda$ where the function should search for $\lambda_{0}$ and consequently $T_{0}$. The value of $T_{0}$ returned by the function was 53 , which is very close to the 51 used to generate the data. The user can also see the value of the norm of $\boldsymbol{\Delta}_{T}$ correspondent to the estimated $T_{0}$.

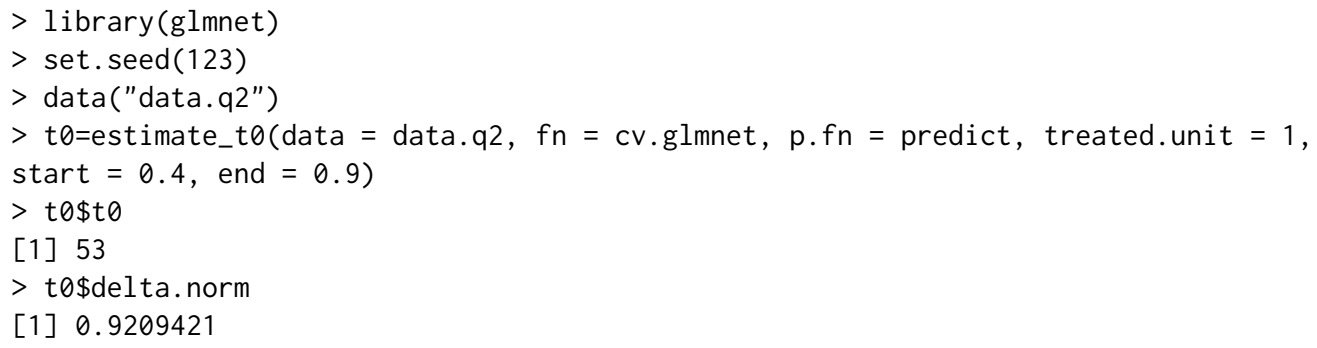

The natural step that follows is to use the estimated $T_{0}$ on the fitArCo function. The results will obviously be very similar to the ones presented on the previous section because the estimated $T_{0}$ is nearly the same as the value used to generate the data.

\footnotetext{
${ }^{6}$ OLS should be used only for small problems with considerably more observations than variables in the period before the intervention.
} 


\section{Application to Real Data}

This sections shows the ArCo package working on two different examples with real data. The first example is from Carvalho et al. (2016b) and it shows the effects on inflation of an tax evasion program on the Brazilian metropolitan area of São Paulo. The second example uses data from the package Synth (Abadie et al., 2011) to compare the ArCo with the Synthetic Control (Abadie and Gardeazabal, 2003; Abadie et al., 2010).

So far we have used OLS and cross-validation LASSO to make the examples as simple as possible. However, there are some advantages on selecting the LASSO regularization parameter using information criterion such as the Bayesian Information Criterion (BIC), which allow us to consistently estimate the degrees of freedom of the model (Zou et al., 2007). Moreover, choosing by information criterion is faster and avoids further complications created by cross-validation on time-series. For the next examples we will define the functions $\mathrm{fn}$ and $\mathrm{p}$. $\mathrm{fn}$ to to select the model using the BIC as follows:

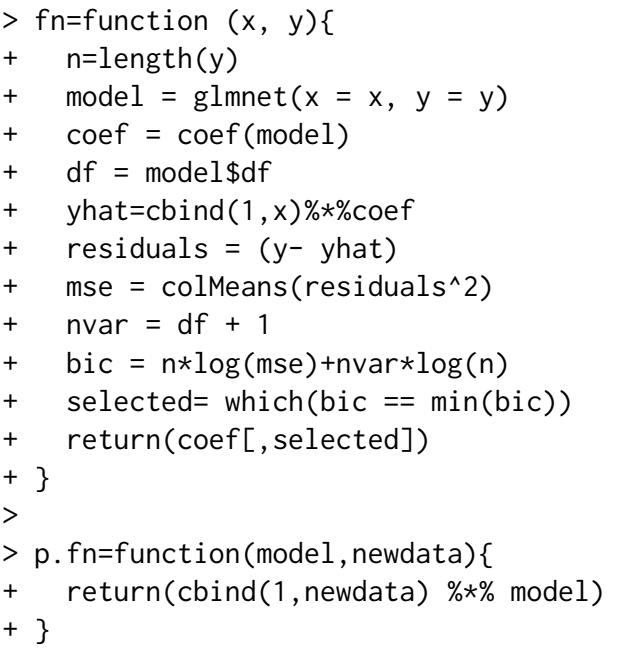

\section{The Effects of an Anti Tax Evasion Program}

In October 2007 the government of the Brazilian state of São Paulo launched an anti tax evasion program called Nota Fiscal Paulista (NFP). It consists of a refund from a state tax on the circulation of products and services. The NFP acts as an incentive for the consumer to ask for electronic sales receipt. The receipts give the consumer the right to participate on monthly lotteries promoted by the government and the tax rebate if his tax identifier number (CPF) is included in the receipt.

The NFP program received substantial support from the population. In January 2008, 413 thousand people were already registered in the program while in October 2013 this number of participants were more than 15 million.

The problem arises if we assume that there was some degree of tax evasion occurring before the intervention, and once the consumers start demanding the receipts, the sellers were forced to pay more tax and evasion became more difficult. If the sellers have some degree of market power they might increase prices in order to accommodate the extra tax. If these assumptions are true one would expect an upward movement on prices due to an increase on marginal costs. Therefore, the example presented in this section tries to investigate whether the NFP had and impact on consumers price in São Paulo.

The first sector to have the NFP was restaurants, therefore the Food Away from Home component (FAH) of the Consumer Prices Index (CPI) is a good measure to contemplate the entire program from the start. The sample is available on the ArCo package with the name of inflationNFP. It consists of nine Brazilian metropolitan areas including São Paulo from January 1995 to September 2009. Each metropolitan area has the interpretation of an ArCo unit. The variables are the FAH component of the CPI inflation and the monthly GDP growth for all metropolitan areas. The treatment happened at $T_{0}=34$, therefore, we have 33 observations to estimate the first step of the ArCo and 23 observations to calculate the counterfactual.

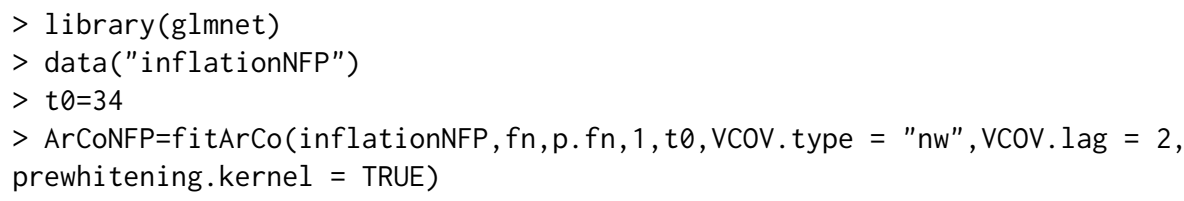


$>\operatorname{plot}($ ArCoNFP, plot=1, display.fitted $=$ TRUE $)$

$>$ ArCoNFP\$delta

$\begin{array}{lrrr} & \text { LB } & \text { delta } & \text { UB } \\ \text { inflationFAH } & 0.226033437 & 0.4226199609 & 0.619206484 \\ \text { GDP } & -0.004699182 & -0.0006055599 & 0.003488062 \\ >\text { ArCoNFP\$p. value } & & \\ \text { Joint inflationFAH } & \text { GDP } & \text { in } \\ \text { 1.333664e-04 } & 2.514289 \mathrm{e}-05 & 7.718676 \mathrm{e}-01 & \end{array}$

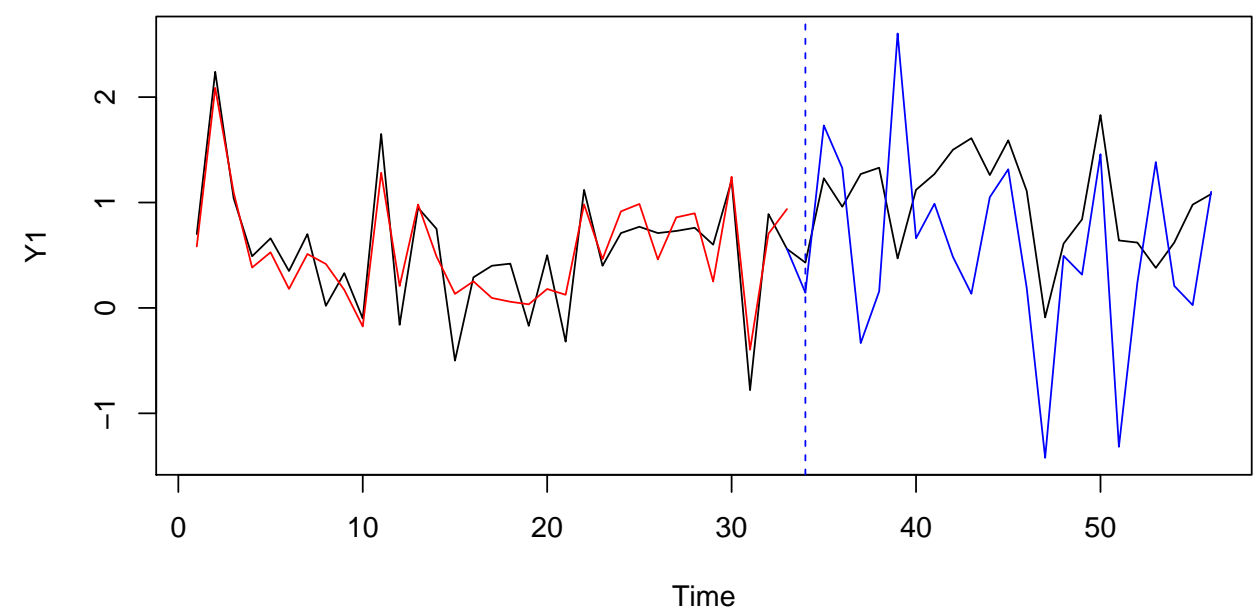

- Observed

Figure 4: plot of the counterfactual of the CPI Inflation (FAH) from São Paulo, Brazil.

The $\$$ delta shows that in fact there was a significant impact on inflation caused by the NFP. The impact on the GPD was statistically zero. The $\$ p$. value relates to an overall effect considering all variables, and it also shows that the effects of the NFP were, in general, different from zero. Figure 4 shows that the LASSO had a good fit in sample, but not too good for us to be concerned with over-fitting. In nearly all periods the estimated counterfactual was below the realized value of the inflation after the NFP was introduced.

It is also interesting to see what happened with the CPI. The code below accumulates the realized inflation and the counterfactual. Figure 5 shows that the accumulated increase on the CPI (FAH) might have been significantly smaller without the NFP.

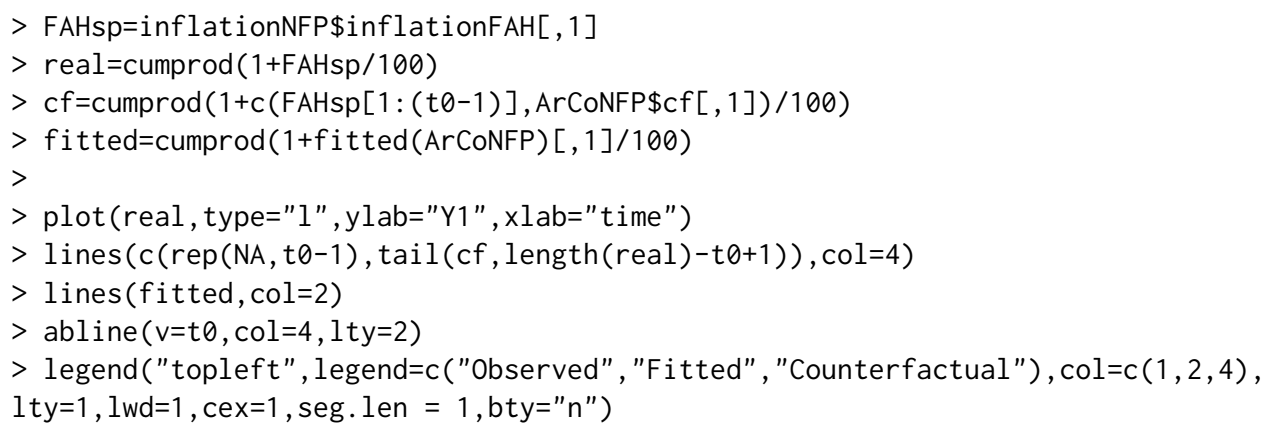

\section{A Comparison with Synthetic Control}

The package Synth provides a user friendly implementation of the SC method and some datasets to test it. In this section we compare the SC and the ArCo by reproducing the exact examples in the synth 


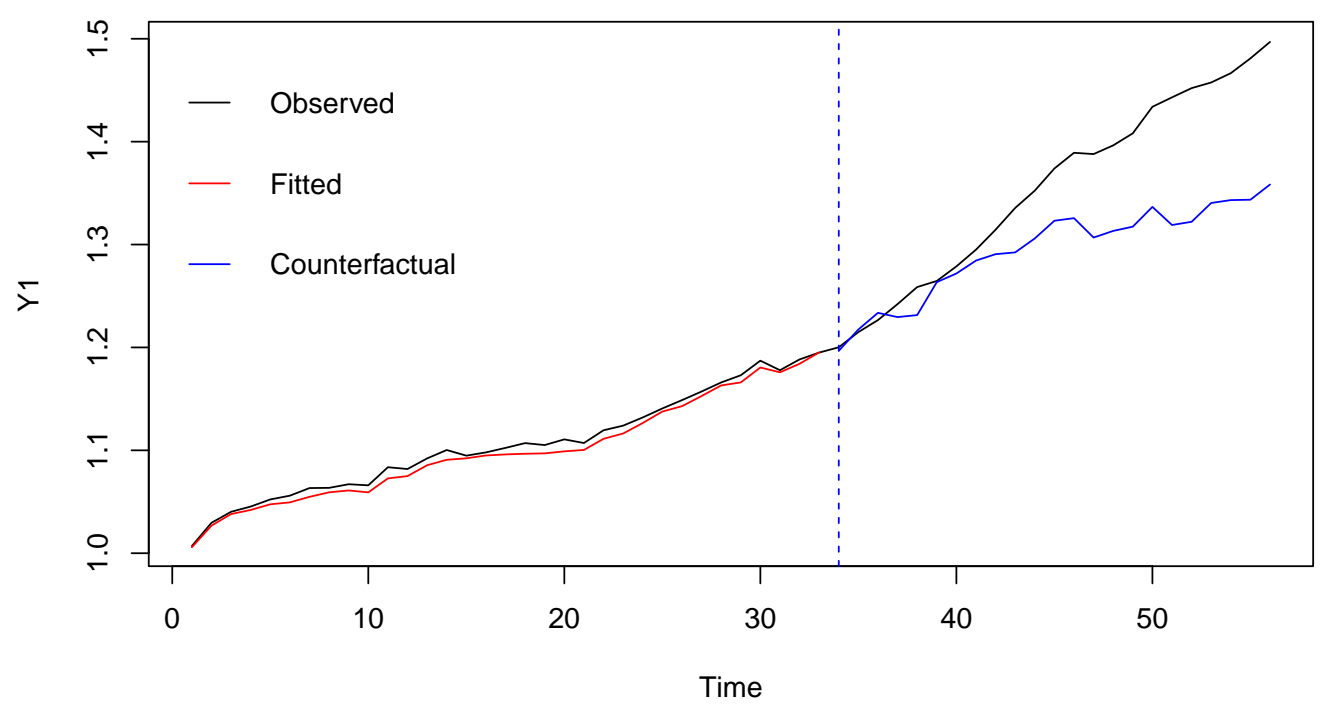

Figure 5: plot of the counterfactual of the CPI (FAH) from São Paulo, Brazil.

function from the Synth package. The first example is with generate data, the exact code is reproduced below. The variable of interest is $Y$ and there are three controls. We can only use the control $X_{2}$ in the ArCo because $X_{1}$ and $X_{3}$ do not have enough observations. A crucial difference between the ArCo and the SC is that the second kills the time dimension, and therefore, require less data. The ArCo uses the entire temporal information and needs all the time-series to be complete. Therefore, we dropped the variables with missing values in the ArCo estimation for the next two examples. There is no built-in NA treatment in the ArCo package because it is as particular feature of each time-series problem. If the user is dealing with data in different periodicities, for example, monthly and quarterly, he/she could repeat the quarterly data in the months with NA or collapse the monthly data to quarterly data.

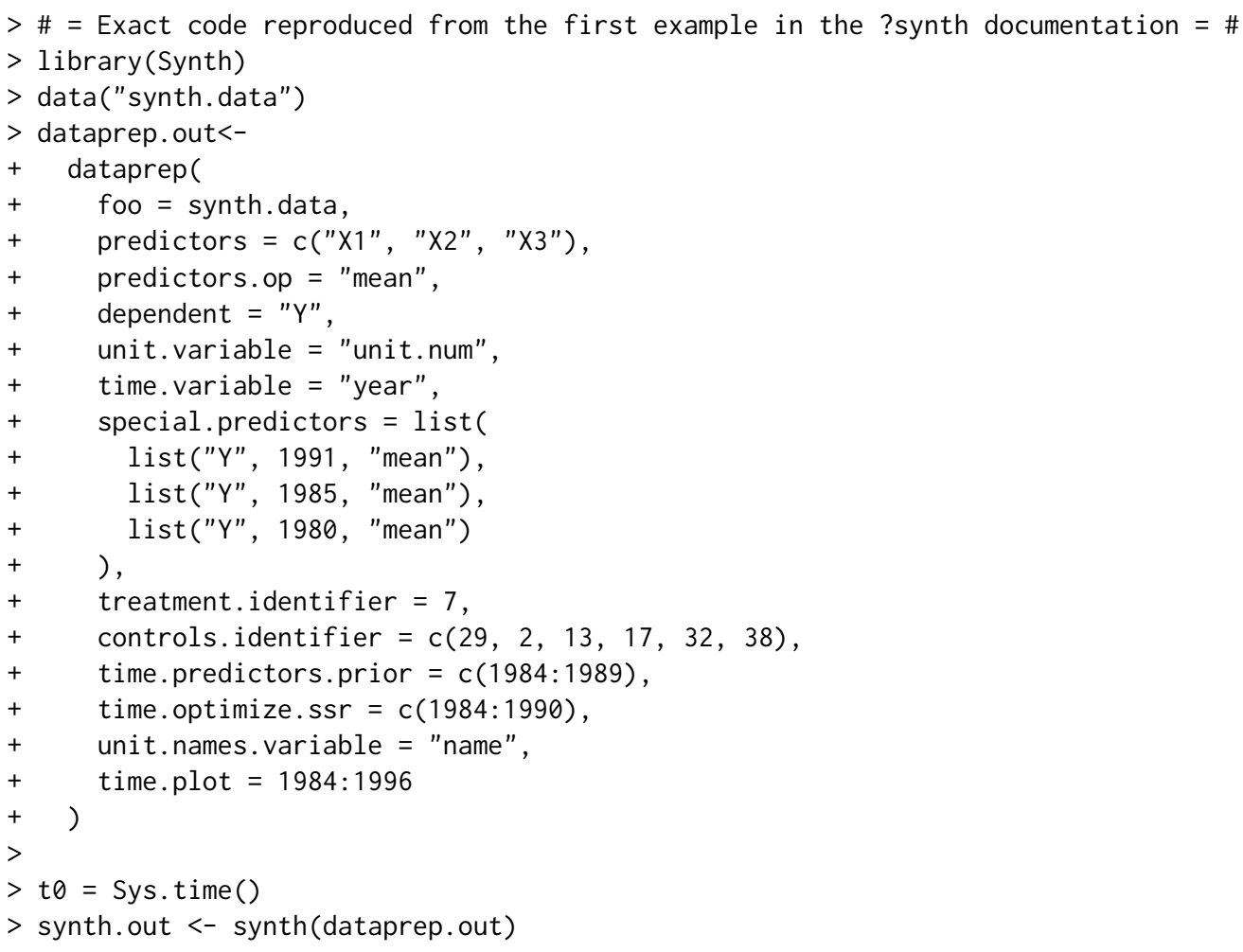


$\mathrm{X} 1, \mathrm{X} 0, \mathrm{Z1}, \mathrm{Z0}$ all come directly from dataprep object.

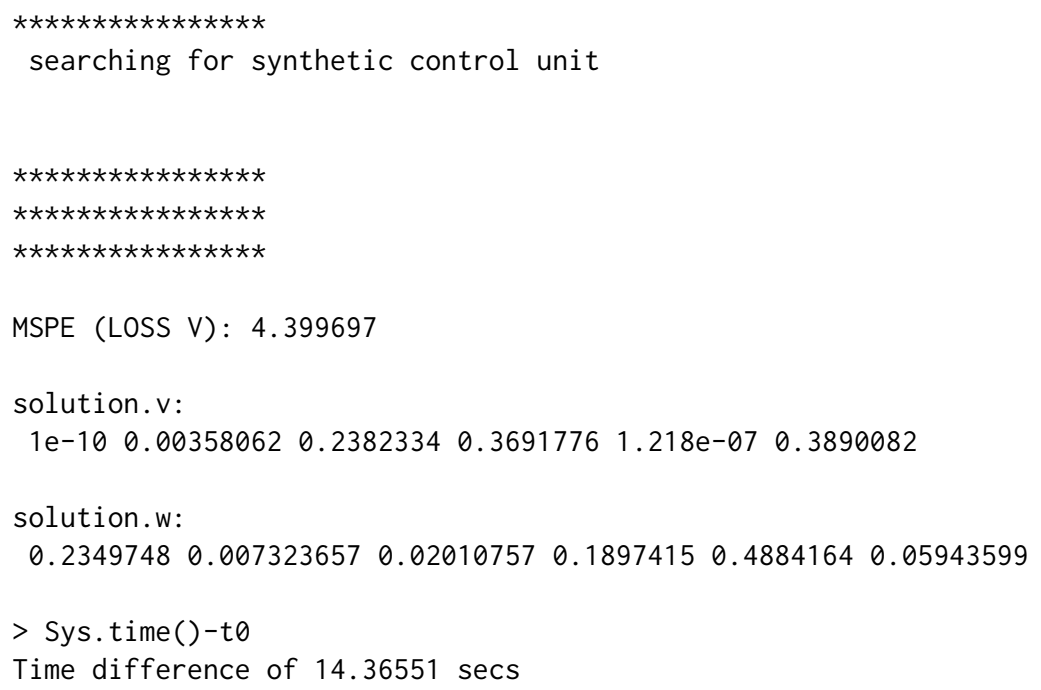

The synth. data is a panel with variables for the time and the units. We can transform it into a list using the function panel_to_ArCo_list. The code below shows the structure of the data, its transformation into list and runs the ArCo using LASSO selecting the best model using the BIC.

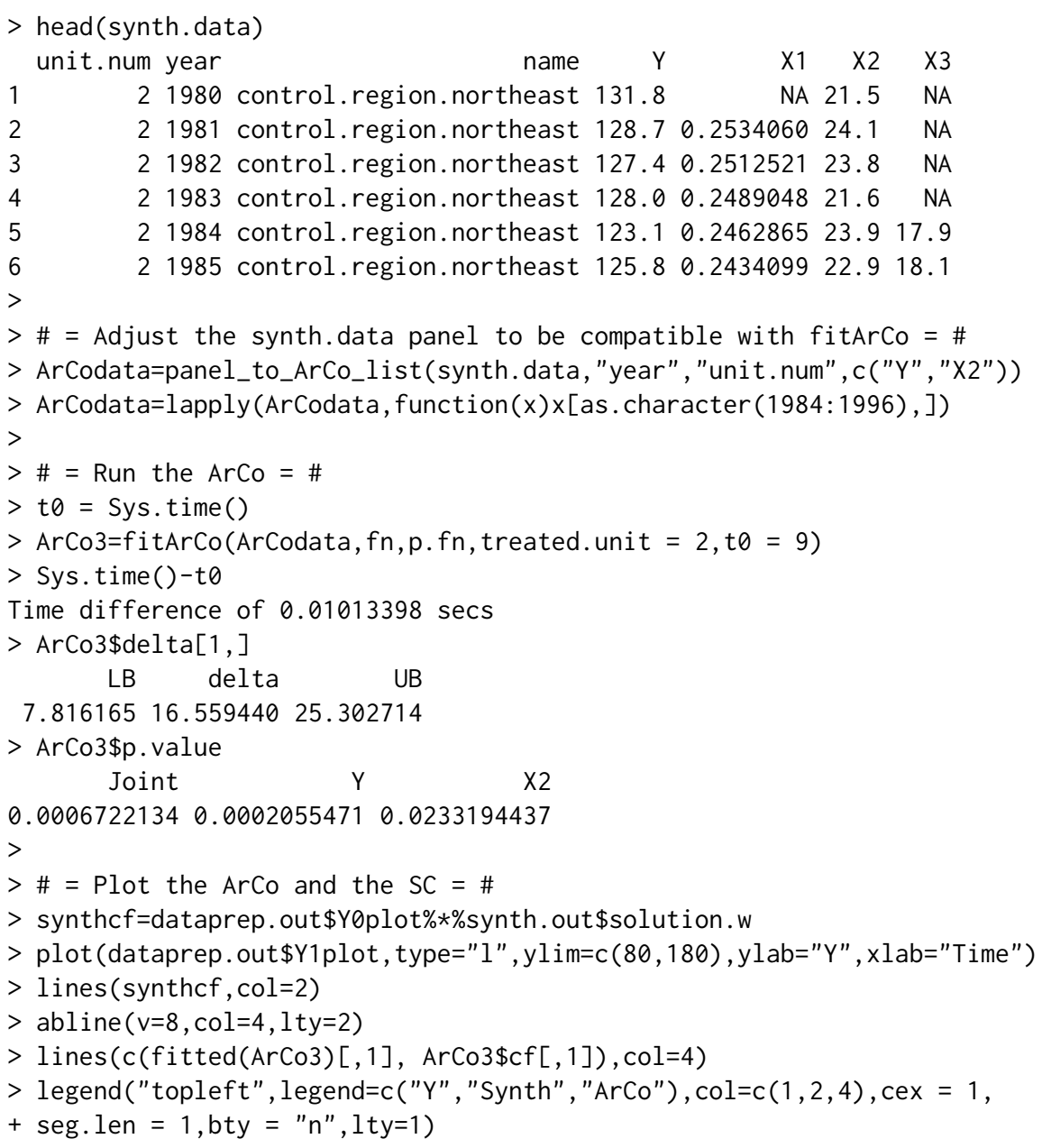

The ArCo took 0.01 seconds to run by LASSO, while the SC took 14 seconds. Figure 6 shows that the two methods had similar results. We used 1992 as the first treated observation $\left(T_{0}\right)$ in the ArCo.The in-sample and out-of-sample are plotted in the same line. Note that we had to drop two variables in the ArCo because of missing values.

The second example in the Synth package is to estimate the counterfactual of the GDP in the 


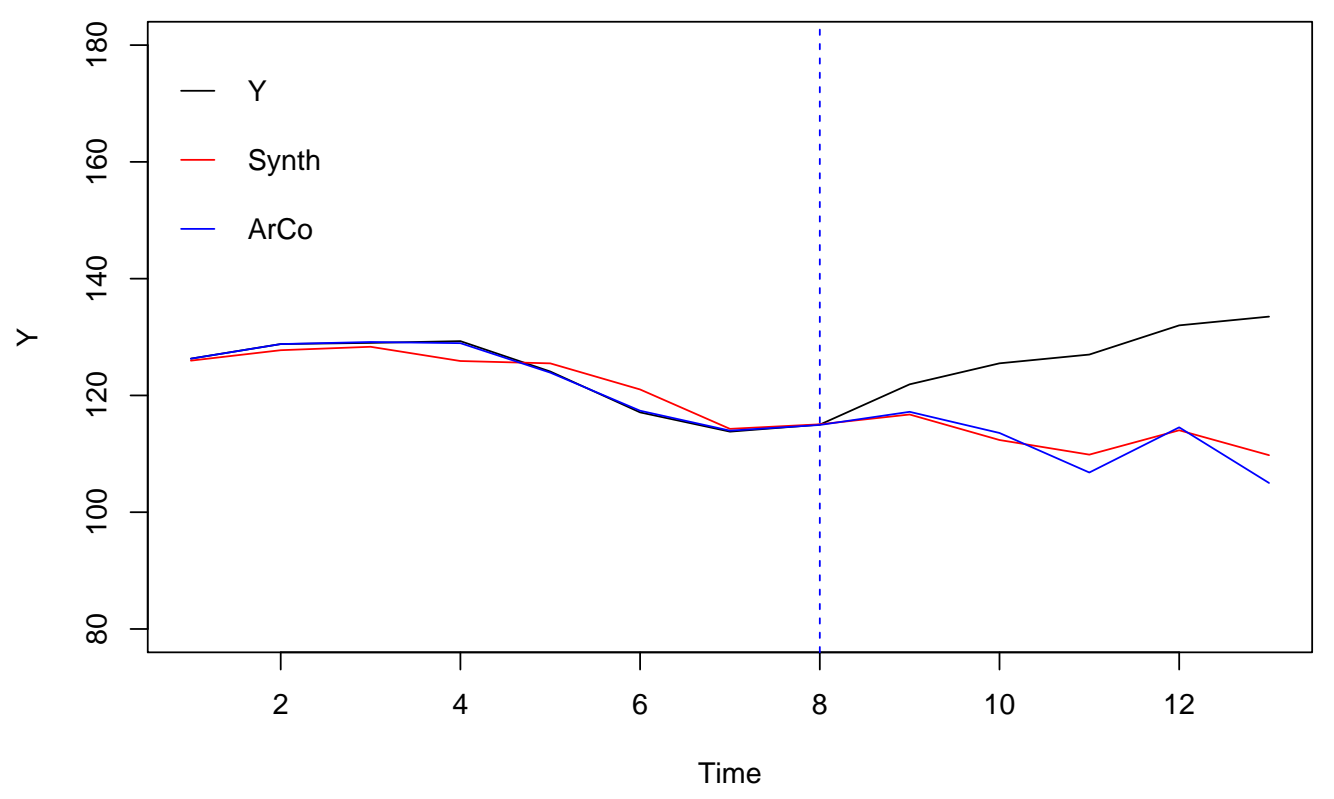

Figure 6: ArCo and Synthetic Control using the data from the first example from the synth function

Basque using other Spanish regions as controls. The main assumption is that the terrorism in the 70s had a negative impact on GDP. The dataset has GDP, investment and several other variables but we can only use the first two because of missing observations. Most of the other variables are related to sector production and education. For more details use ?basque.

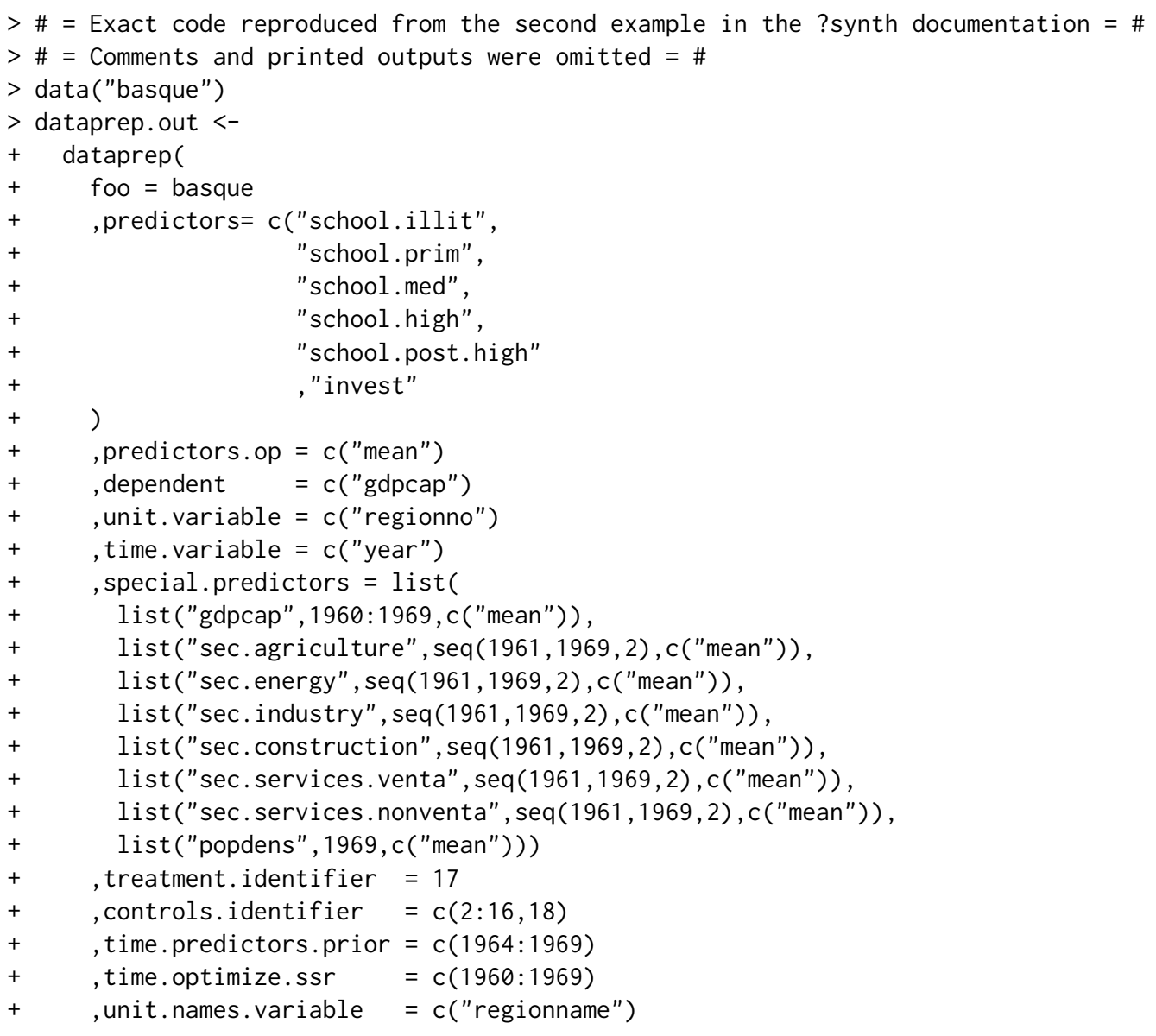




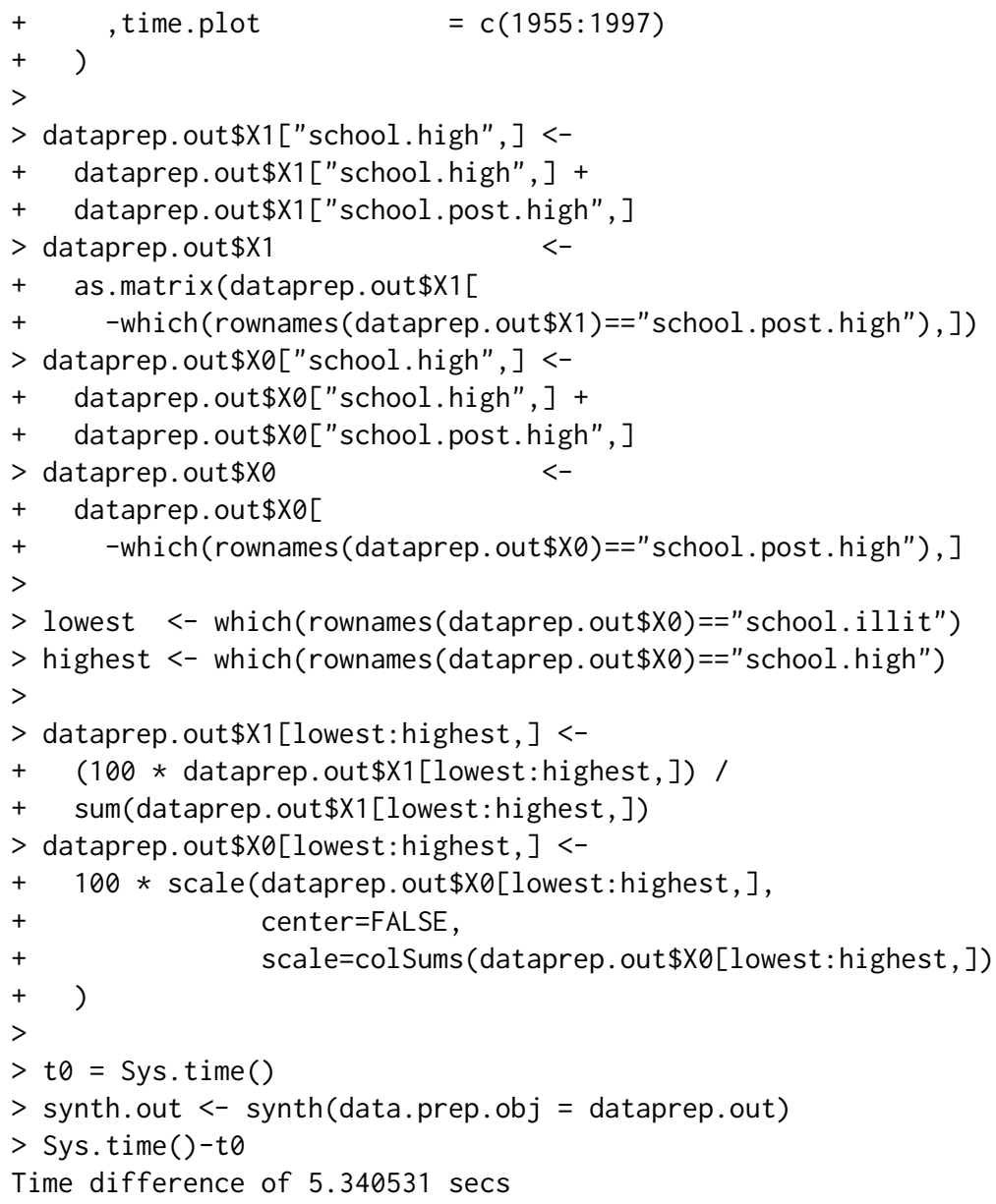

Since the GDP is non-stationary, it was first differentiated to estimate the ArCo. The code below prepares the data, which also comes in a panel, estimates the ArCo using LASSO and the BIC to select the best model and rebuild the GDP on its original level to compare with the SC. Since we do not know exactly when in the 70s the effects on the GDP begun, we also estimated $T_{0}$ to be used in the ArCo.

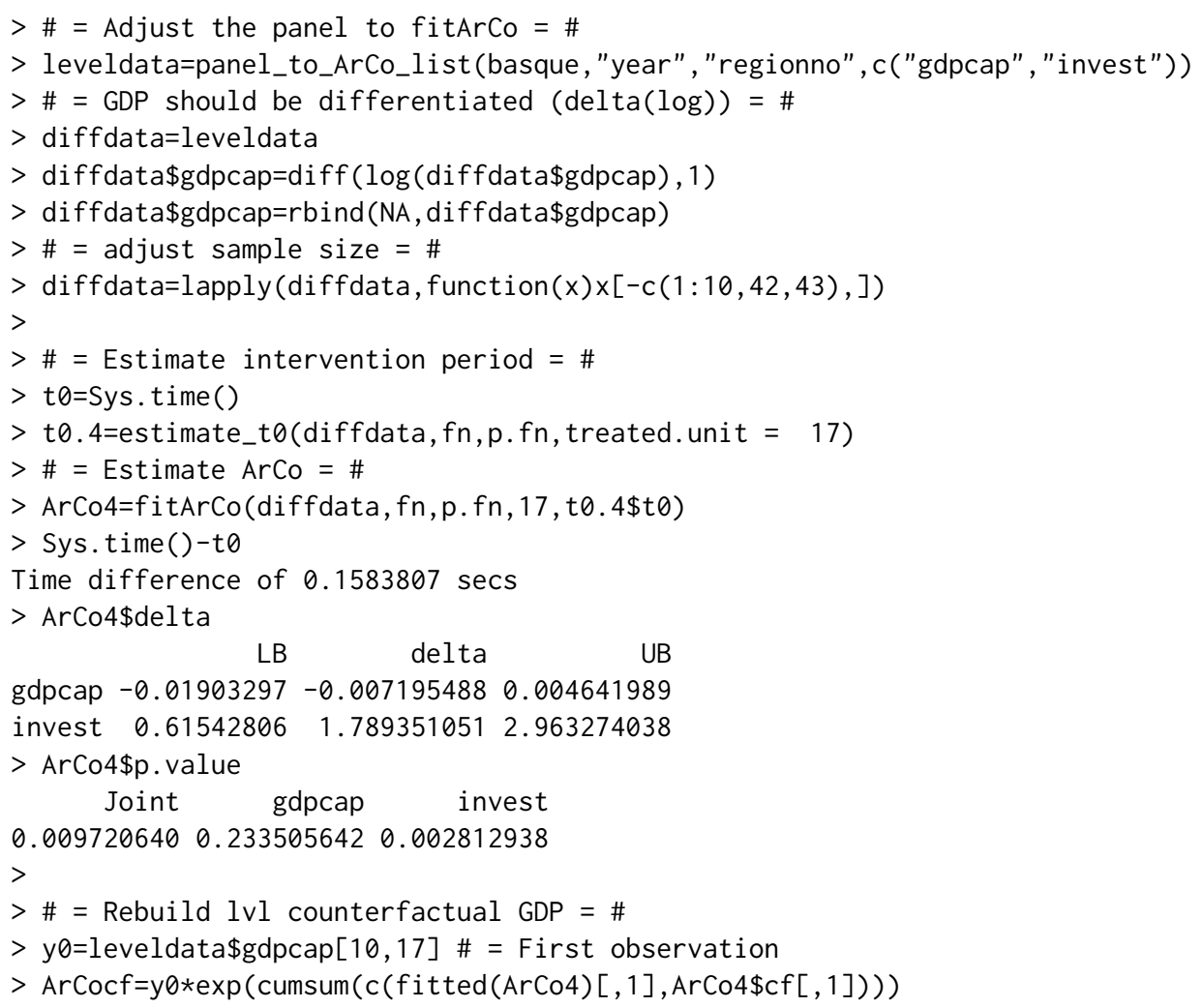




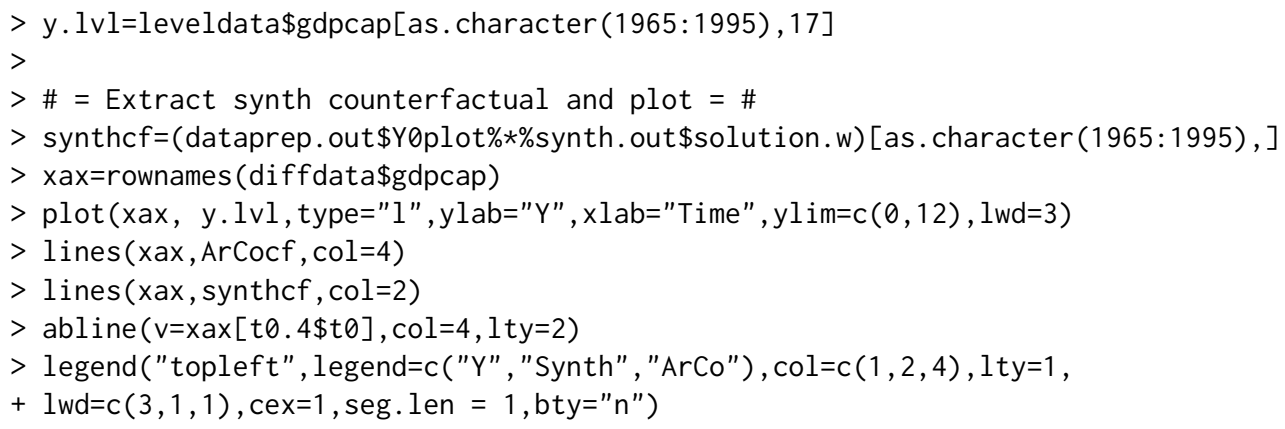

The \$delta showed that the intervention was not significant for the differentiated GDP, however, Figure 7 shows that there was a considerable impact on its level. The difference estimated by the ArCo is even bigger than the one estimated by the SC. It also seems that the ArCo counterfactual follows the trend before the intervention while the SC simply shifts the observed trend after the intervention.

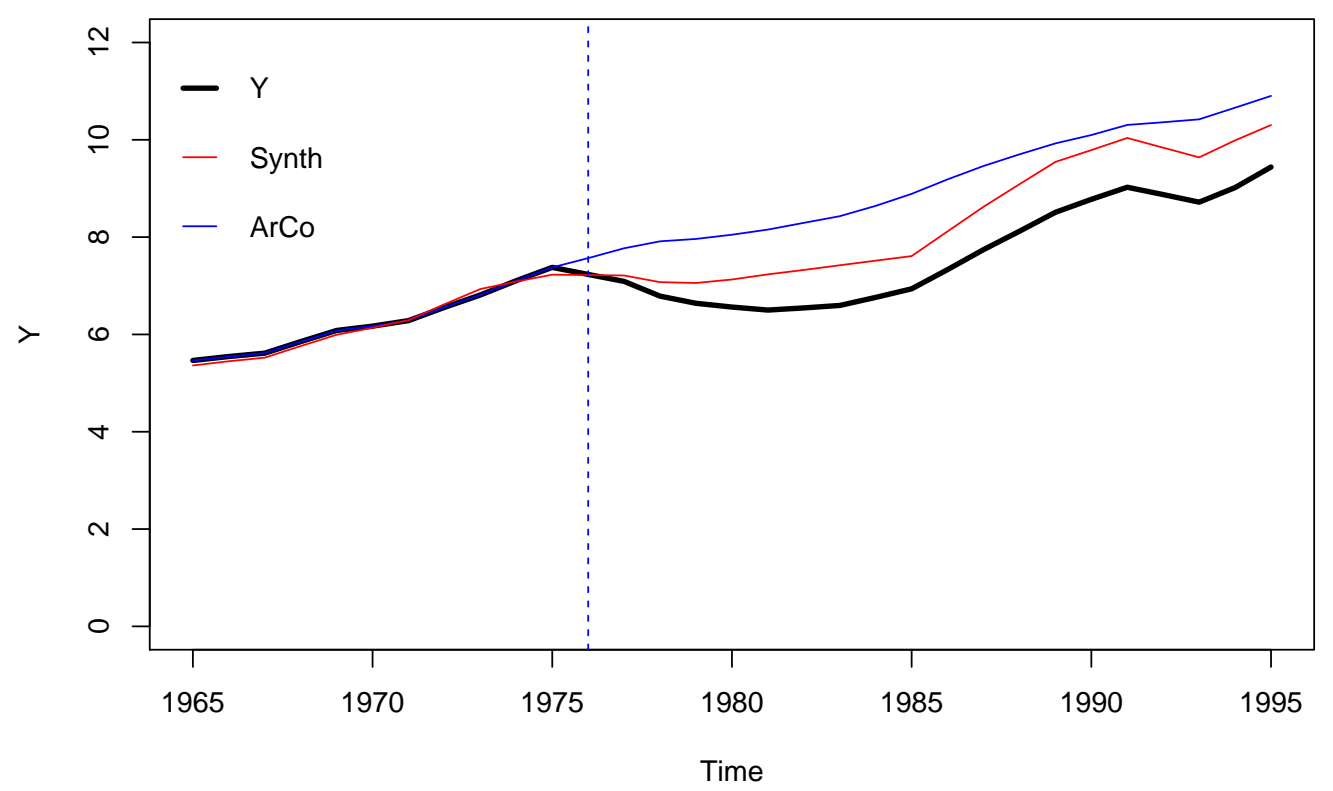

Figure 7: ArCo and Synthetic Control using the data from the Basque example from the synth function

\section{Final Remarks}

In this paper we have introduced the ArCo package which consists in a set of R functions implementing the ArCo method proposed by Carvalho et al. (2016b), which is a generalization of the Synthetic Control of Abadie and Gardeazabal (2003) and Abadie et al. (2010) and the panel approach put forward by Hsiao et al. (2012).

The ArCo method is a two-step methodology where in the first step a counterfactual is estimated based on a large, possibly high-dimensional, panel of time series from untreated peers. The package is very flexible and the user can choose any model of her preference such as, for example, LASSO regressions, random forests, boosted trees, neural networks, among others. In the second stage the average treatment effect is computed and a test for the null of no intervention effect is provided.

We believe that this $\mathrm{R}$ package will serve as an useful addition to the econometrics toolbox to conduct counterfactual analysis in comparative case studies.

\section{Acknowledgements}

We would like to thank an anonymous referee for very helpful and detailed comments. 


\section{Bibliography}

A. Abadie and J. Gardeazabal. The economic costs of conflict: A case study of the Basque country. American Economic Review, 93:113-132, 2003. [p91, 92, 100, 106]

A. Abadie, A. Diamond, and J. Hainmueller. Synthetic control methods for comparative case studies: Estimating the effect of California's tobacco control program. Journal of the American statistical Association, 105:493-505, 2010. [p100, 106]

A. Abadie, A. Diamond, and J. Hainmueller. Synth: An R package for synthetic control methods in comparative case studies. Journal of Statistical Software, 42(13):1-17, 2011. [p91, 100]

D. W. K. Andrews and C. J. Monahan. An improved heteroskedasticity and autocorrelation consistent covariance matrix estimator. Econometrica, pages 953-966, 1992. [p94, 97]

S. Athey and G. W. Imbens. The state of applied econometrics - causality and policy evaluation. Technical report, arXiv:1607.00699v1, 2016a. [p91]

S. Athey and G. W. Imbens. The econometrics of randomized experiments. 1607.00698, arXiv, $2016 \mathrm{~b}$. [p91]

A. Canty and B. D. Ripley. boot: Bootstrap R (S-Plus) Functions, 2015. URL https: //cran. r-project. org/web/packages/boot/. R package version 1.3-17. [p97]

C. V. Carvalho, R. P. Masini, and M. C. Medeiros. The perils of counterfactual analysis with integrated processes. Working paper, Pontifical Catholic University of Rio de Janeiro, 2016a. Available at SSRN: https: / / ssrn.com/abstract=2894065 or http:/ / dx.doi.org/10.2139/ssrn.2894065. [p92, 93]

C. V. Carvalho, R. P. Masini, and M. C. Medeiros. ArCo: An artificial counterfactual approach for high-dimensional panel time-series data. Working paper, Pontifical Catholic University of Rio de Janeiro, 2016b. Available at SSRN: https://ssrn.com/abstract=2823687 or http:/ /dx.doi.org/10.2139/ssrn.2823687. [p92, 93, 94, 95, 100, 106]

N. Doudchenko and G. W. Imbens. Balancing, regression, difference-in-differences and synthetic control methods: A synthesis. 22791, NBER, 2016. [p92, 93]

B. Ferman and C. Pinto. Revisiting the synthetic control estimator. Working paper, São Paulo School of Economics - FGV, 2016. [p92]

B. Ferman, C. Pinto, and V. Possebom. Cherry picking with synthetic controls. Working paper, São Paulo School of Economics - FGV, 2016. [p93]

Y. R. Fonseca, M. C. Medeiros, R. P. Masini, and G. F. R. Vasconcelos. ArCo: Artificial Counterfactual Package, 2017. URL https://CRAN. R-project. org/package=ArCo. R package version 0.1-2. [p93]

J. Friedman, T. Hastie, and R. Tibshirani. Regularization paths for generalized linear models via coordinate descent. Journal of Statistical Software, 33:1-22, 2010. [p97]

L. Gobillon and T. Magnac. Regional policy evaluation: Interactive fixed effects and synthetic controls. Review of Economics and Statistics, 98:535-551, 2016. [p92]

C. Hsiao, H. S. Ching, and S. K. Wan. A panel data approach for program evaluation: Measuring the benefits of political and economic integration of Hong Kong with mainland China. Journal of Applied Econometrics, 27:705-740, 2012. [p91, 92, 106]

W. Newey and K. West. A simple, positive semi-definite, heteroskedasticity and autocorrelation consistent covariance matrix. Econometrica, 55:703-08, 1987. [p94, 97]

R. Tibshirani. Regression shrinkage and selection via the LASSO. Journal of the Royal Statistical Society. Series B (Methodological), pages 267-288, 1996. [p92]

H. Zou, T. Hastie, and R. Tibshirani. On the degrees of freedom of the Lasso. The Annals of Statistics, 35:2173-2192, 2007. [p100]

Yuri R. Fonseca

Department of Materials Science, Military Institute of Engineering (IME)

Praça Gen Tibúrcio 80, Praia Vermelha, Rio de Janeiro, RJ

Brazil

yuriresendefonseca@outlook.com 
Ricardo P. Masini

São Paulo School of Economics - FGV-SP

Address Rua Itapeva 474, Bela Vista, São Paulo, SP

Brazil

ricardo.masini@fgv.br

Marcelo C. Medeiros

Department of Economics, Pontifical Catholic University of Rio de Janeiro (PUC-Rio)

Rua Marquês de São Vicente 225, Gávea, Rio de Janeiro, RJ

Brazil

mcmQecon. puc-rio.br

Gabriel F. R. Vasconcelos

Department of Electrical Engineering, Pontifical Catholic University of Rio de Janeiro (PUC-Rio)

Rua Marquês de São Vicente 225, Gávea, Rio de Janeiro, RJ

Brazil

gabrielrvsc@yahoo.com.br 